\title{
Design of an Online Monitoring System of Lithium Ion Energy Storage Batteries for a Distributed Power Station
}

\author{
https://doi.org/10.3991/ijoe.v14i12.9402 \\ Zhai Haizhou \\ Heze University, Heze, China \\ kateng0204@126.com
}

\begin{abstract}
Aiming at the online monitoring of real-time operating of lithiumion energy storage batteries for distributed power station, this paper studies the online monitoring system of lithium-ion energy storage batteries based on $\mathrm{B} / \mathrm{S}$ network structure. The system consists of a battery status information collection unit, a centralized control unit, and a remote monitoring center. The battery status information collection unit detects the current, voltage, temperature and other parameters of the lithium ion energy storage battery in real time, transmits the data to the centralized control unit for data processing and real-time estimation of the SOC of the battery, and then the centralized control unit transmits the measurement parameters through the Internet network. The remote monitoring center monitors and analyzes the battery real-time status data, accurately calculates the battery SOC, realizes the online real-time monitoring of the lithium ion battery, prevents the lithium ion battery from overcharging, over-discharging, overheating, and promotes the safe and stable operation of the lithium-ion energy storage battery.
\end{abstract}

Keywords—online monitoring system, lithium-ion battery, distributed power station

\section{Introduction}

As a green energy, distributed power generation technology has attracted more and more attention[1-3]. Nowadays the new energy generation methods such as solar energy are progressing rapidly[2,3]. However, distributed power generation such as solar power generation and wind power has its own volatility and is greatly affected by external factors such as light and temperature.

The volatility and dispersion characteristics of distributed generation require energy storage batteries to stabilize fluctuations, match supply and demand, and improve power supply quality[2,3]. Distributed battery energy storage system can weaken or even eliminate the fluctuation of new energy power generation and enhance the stability of new energy power generation. It is the core equipment to achieve dynamic matching between power generation curve and power consumption curve. Many countries all over the world are competing to develop distributed energy storage. 
The performance and state of energy storage batteries have a decisive influence on the stable, safe, reliable, long-life, and low-cost operation of distributed generation. In distributed battery energy storage, lithium-ion battery energy storage mode has the advantages of fast effect rate, high energy conversion efficiency, fast cost reduction and good scalability[4,5]. Therefore, lithium battery energy storage is one of the most energy storage methods for market applications. However, the efficiency, operation and maintenance cost, safety and reliability of a large number of energy storage battery cells are currently recognized as technical problems in the world. The charging/discharging of battery is an electrochemical reaction process, and its working process exhibits very strong nonlinear characteristics[4-6].

Online monitoring on the state of energy storage batteries is very important. The implementation of the online monitoring system provides a powerful tool for people to obtain the operating status of the battery pack anytime and anywhere[7-9]. With the long-term use of the online monitoring system, the regular maintenance of the battery can be gradually converted into active maintenance with reducing maintenance risks and maintenance costs.

\section{Lithium ion energy storage battery}

The energy storage battery of the distributed power station uses a lithium ion battery. Lithium-ion undergos directional motion in the positive and negative electrodes and the electrolyte membrane during charging or discharging. In a distributed power station, lithium-ion energy storage batteries are used in series and in parallel, and can be flexibly combined into a plurality of battery packs, as shown in Figure 1.
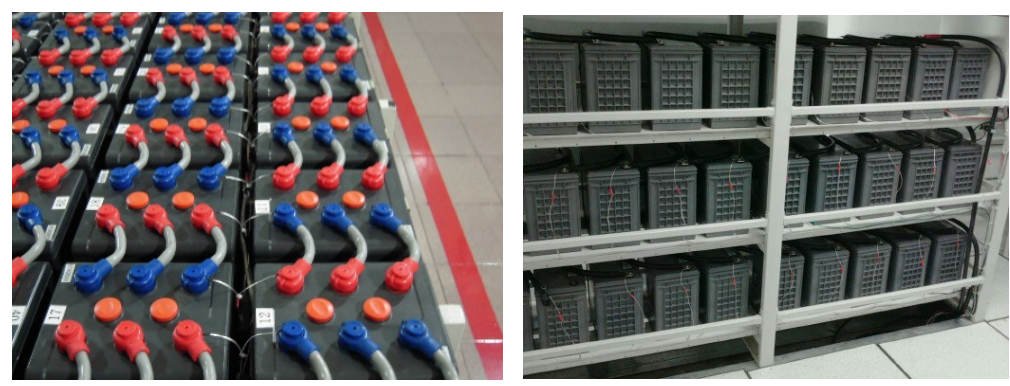

Fig. 1. The different ways of energy storage battery grouping

\subsection{Principle of electrochemical reaction of lithium ion battery}

Lithium-ion battery is mainly composed of two electrodes, electrolytes and porous partitions[1,4-6]. The active material lithium ion is provided by a lithium-containing transition metal oxide such as $\mathrm{LiCoO} 2, \mathrm{LiNiO} 2, \mathrm{LiMn} 2 \mathrm{O} 4$ in positive electrode. The working material for lithium ions embedding in is coke, graphite, amorphous carbon and so on in negative electrode. Electrolyte is dissolved in the lithium salt of organic 
solvents. The diaphragm is porous, electrically insulated, and is physical barriers between the poles that allow ions to pass through. Lithium-ion batteries comply with the "rocking chair" principle in the charging and discharging process. When charging, Li + leaving the positive layer of the layered structure, under the action of external voltage, through the electrolyte, porous separator, migrate to the underlying structure of the negative, and the electrons flow from the positive to the negative through external wire. At the time of discharging, $\mathrm{Li}+$ leaves the negative layer of the lamellar structure, through the porous separator, migrating to the positive structure of the layered structure, and electrons flow in external circuit to form the current, so the chemical energy conversion is achieved. The electrochemical reactions occurring at the positive and negative electrodes are:

Positive reaction :

$$
\begin{gathered}
\mathrm{Li}_{1-x} \mathrm{MO}_{2}+x \mathrm{Li}^{+}+x e^{-} \stackrel{\text { discharging }}{\longrightarrow} \mathrm{LiMO}_{2} \\
\mathrm{LiMO}_{2} \stackrel{\text { charging }}{\longrightarrow} \mathrm{Li}_{1-x} \mathrm{MO}_{2}+x \mathrm{Li}^{+}+x e^{-}
\end{gathered}
$$

Negative reaction :

$$
\begin{gathered}
L i_{x} C_{6} \stackrel{\text { discharging }}{\longrightarrow} x \mathrm{Li}^{+}+x e^{-}+C_{6} \\
\mathrm{Li}^{+}+x \mathrm{e}^{-}+\mathrm{C}_{6} \stackrel{\text { charging }}{\longrightarrow} x \mathrm{Li}_{x} \mathrm{C}_{6}
\end{gathered}
$$

\subsection{Lithium-ion battery equivalent model}

For system-level development and control applications, it is preferred use of the equivalent circuit model on battery research. Engineering uses the equivalent circuit model to model the thermal-electrical characteristics of battery, with parametric techniques that combine the model and experimental measurements to optimize the nonlinear elements of the equivalent circuit. In this paper, the two-order RC equivalent circuit model is used as the circuit model of lithium ion battery with simplicity and good realtime performance.

The 2RC equivalent circuit model of Li-ion battery is established on basic of the Thevenin model which has been extended with several new capacitors and resistors, and the 2RC equivalent circuit model is shown in Fig. 2. Resistive and capacitive characteristics of the battery mainly capacitive impedance raised from polarization phenomena and ohmic resistance of the battery as stated in $[1,6]$. The capacitive impedance produced from polarization phenomena can be simulated with the resistor $\mathrm{R}$ and the capacitor $\mathrm{C}$.

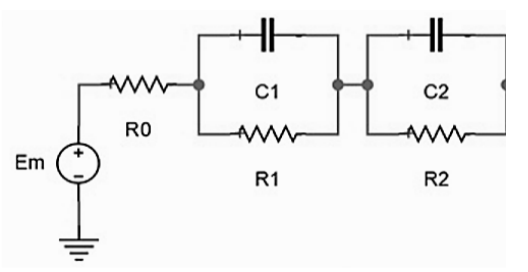

Fig. 2. The $2 \mathrm{RC}$ equivalent circuit model with two time constant, internal resistance and open circuit voltage 
In the Thevenin 2RC equivalent circuit model, the following equations can be obtained:

$$
\begin{gathered}
E_{m}=R_{0} i_{t}+R_{1} i_{r t} \\
i_{t}=i_{c t}+i_{r t} \\
i_{c t} / C_{1}=R_{1} \frac{d i_{r t}}{d t}
\end{gathered}
$$

Where $E_{m}$ is the open circuit voltage of battery, $i_{t}$ is the operating current (when charging it is positive, and when discharging it is negative). Where R0 is the internal ohmic resistance of the battery, R1, R2 and $\mathrm{C} 1, \mathrm{C} 2$ are the polarization resistance and the polarization capacitance, both of which form the $\mathrm{RC}$ circuit to describe the polarization of the battery.

\section{$3 \quad$ The structure and working principle of energy storage battery online monitoring system}

\subsection{The function of the energy storage battery online monitoring system}

The online monitoring system is an intelligent system for online monitoring of energy storage batteries in distributed power stations. It can realize real-time online monitoring of the operating conditions of energy storage battery packs, and monitor the status of the energy storage battery packs and various system parameters.

The online monitoring system can monitor the internal resistance and terminal voltage of the battery in real time, calculate the battery capacity, and manage the monitoring data with the SQL-SEVER database, so that the data can be called and analyzed at any time. When the system finds that the battery is degraded, it can promptly alarm, providing a basis for fine maintenance of the battery pack. The system adopts the B/S structure, which facilitates relevant personnel at all levels to access relevant data at any time within the permitted authority, which is beneficial to improve the management level of battery maintenance technology.

\subsection{The working principle and overall structure of the online monitoring system}

The online monitoring system includes a battery status information collection unit, a centralized control unit, and a remote monitoring center. The overall structure of the online monitoring system is shown in Figure 3.

Each battery status information acquisition/detection unit achieves one set of storage battery monitoring function respectively. The battery status information acquisition unit has powerful functions, data can be collected, stored, upload function, these network programs is very simple. Front-end subsystem battery status information acquisition unit and the centralized control unit together constitute a cell line monitoring system, complete all line monitoring battery. 
The centralized control unit is the core of the online monitoring system, and is responsible for the data processing and data transmission communication of the entire system. The centralized control unit configures the network communication interface, and uploads the monitored data to the remote monitoring center in real time through the internet to realize online monitoring of the battery.

The remote control center is responsible for the unified management and monitoring of the operation of the energy storage system of each distributed power station. The remote monitoring and management system is operated remotely through the remote management application software and database software. The remote monitoring center software adopts the $\mathrm{B} / \mathrm{S}$ structure-based mode. The system administrator can assign the user name, password and access rights to all relevant personnel. These related personnel, as IE users, browse the battery data through the Internet within the specified authority. The remote monitoring center adopts database management and adopts SQLSERVER commercial database to facilitate management, query and call of data of each battery pack.

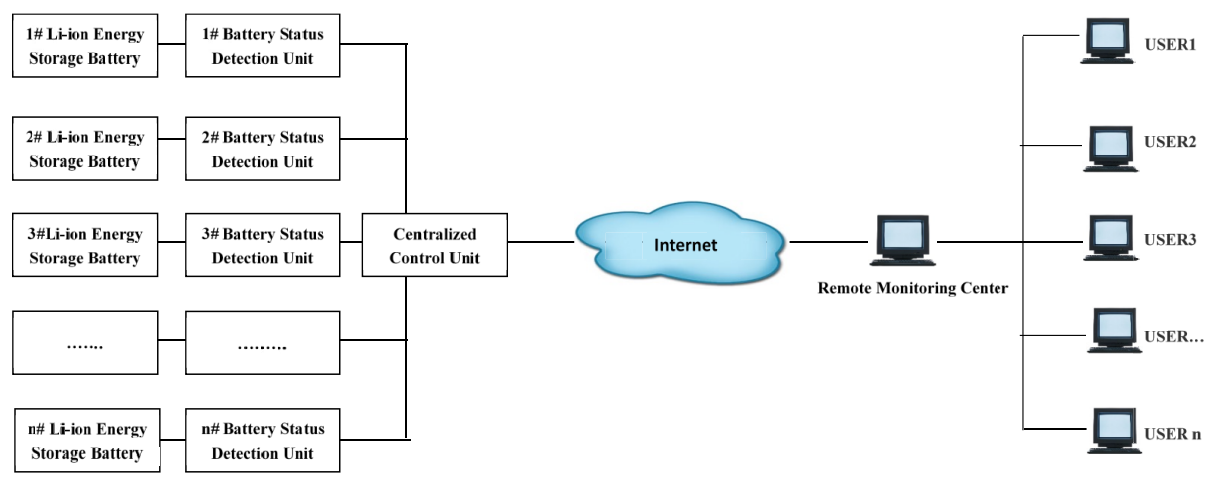

Fig. 3. The overall structure of online monitoring system of energy storage lithium ion battery for distributed power station

\section{$4 \quad$ The battery status information detection unit}

The battery status information detection unit is controlled by a single-chip microcomputer, and is composed of a power supply circuit, a reset circuit, a battery voltage measuring circuit, a battery temperature measuring circuit, and a current measuring circuit.

\subsection{The battery voltage detection circuit}

The battery voltage measuring circuit adopts the technology of multi-channel switching. The multi-channel electronic switch composed of low-impedance MOS tubes divides the voltage signals of the respective cells through voltage division and voltage follower buffering, and then performs A/D conversion to complete measurement of the voltage of each series cell of the lithium ion energy storage battery. A linear 
optocoupler is used as a voltage signal sampling device to collect a single lithium ion battery voltage signal. The battery voltage signal is converted into a current signal and sent to the DSP chip. Then enter the microprocessor through the multiplexer for processing. The lithium ion battery voltage detection circuit is shown in Figure 4.

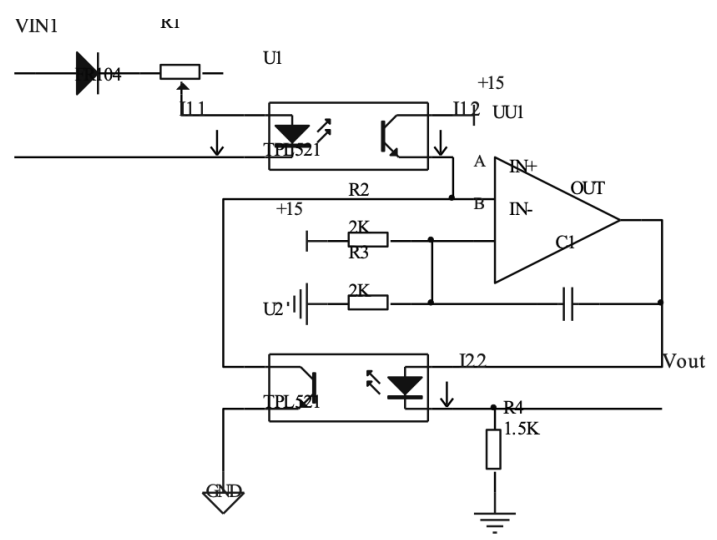

Fig. 4. The diagram of lithium ion single cell voltage detection circuit

The voltage of the single cell (VIN1) is converted into a current signal $\left(\mathrm{I}_{11}\right)$ after the closed loop formed by the variable resistance R1 and the light-emitting diode in the optocoupler $\mathrm{U} 1$, and there is a proportional relationship between $\mathrm{I}_{11}$ and $\mathrm{I}_{12}$. UU1 is used as a comparator in this design. When the voltage $\mathrm{Vb}$ at point $\mathrm{B}$ is less than the voltage Va at point A, UU1 outputs a high voltage; otherwise, UU1 outputs a low voltage. In this circuit, the comparator constitutes a feedback to keep the voltage values of the two points A and B consistent. It can be seen that Vout is proportional to VIN.

\subsection{The battery current detection circuit}

It adopts Swiss LEM DHAB series sensor, which is suitable for measuring DC, AC and pulse current. It is mainly used for high-power, low-voltage battery monitoring and is widely used to measure large battery current. The electrically isolated design between the primary side circuit (high power) and the secondary side circuit (electronic circuit) provides a non-contact, low power solution. The output of the sensor truly reflects the true waveform of the energized conductor. The LEM DHAB output is voltage data with a secondary voltage of $2 \mathrm{~V}$ and the output voltage is input through the LTC6811 auxiliary ADC. It produces two outputs that are proportional to the supplied VCC and produces a connection to the GPIO pin.

\subsection{The battery temperature detection circuit}

Temperature has an important impact on the calculation of the remaining battery power and battery safety. Since the detection of the battery temperature is mainly for the acquisition of the highest and lowest temperatures, an NTC (Negative Temperature 
Coefficient) thermistor is selected as the temperature sensor. The temperature signal acquisition circuit of the single battery is designed, and the thermistor (ie RMDZ1) is used for the temperature detection of the battery. The RMDZ1 is separately mounted on each of the single cells, and combined with the bridge circuit, the temperature signal is converted into a voltage value, thereby completing the measurement of the cell temperature. Because the resistance between NTC thermistor and temperature is a nonlinear function, the software is used to check the full-scale linearization of temperature measurement. The lithium ion cell voltage detection circuit is shown in Figure 5.

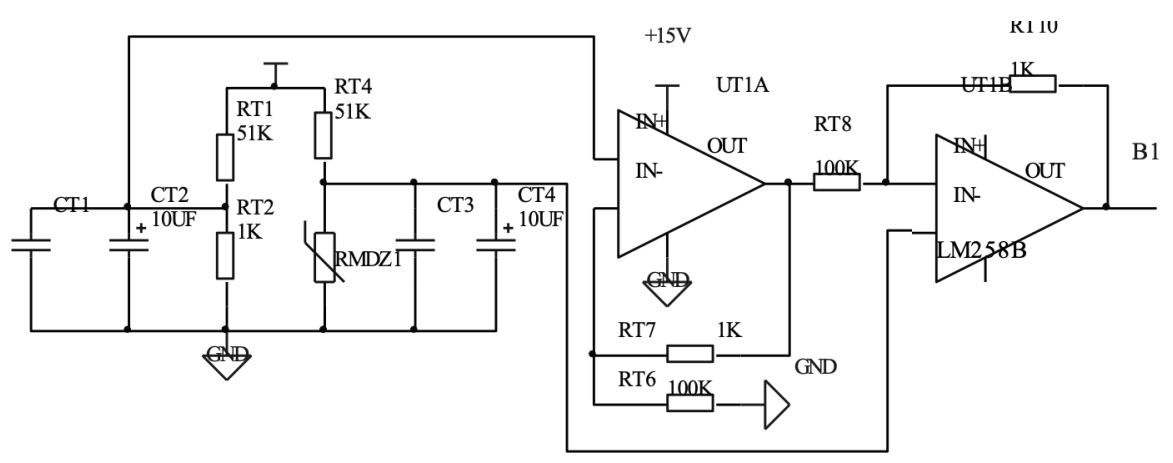

Fig. 5. The diagram of lithium ion single cell temperature detection circuit

\section{The centralized control unit}

The main program of the centralized control unit is programmed in $\mathrm{C}++$. It mainly includes initialization program, communication processing program, real-time operation parameter acquisition (voltage, current and temperature detection), SOC estimation, protection control program, and real-time display program and data management program for lithium ion energy storage battery, as shown in Figure 6.

The centralized control unit is responsible for receiving status information and alarm information sent by the battery status information collection unit, classifying and storing the data, and transmitting the data to the remote monitoring center through the internet network. The centralized control unit communicates with the battery status information collection unit and the remote monitoring center, and provides a visual interface for the user to let the user know whether the battery is working normally. The centralized control unit is modular in design and can be flexibly configured according to the battery status detection unit, as shown in Figure 7(Left picture). The centralized control unit has the real-time visual interface which has a powerful data display function, and can real-time display the operating parameters of lithium ion energy storage battery, as shown in Figure 7(Right picture). 


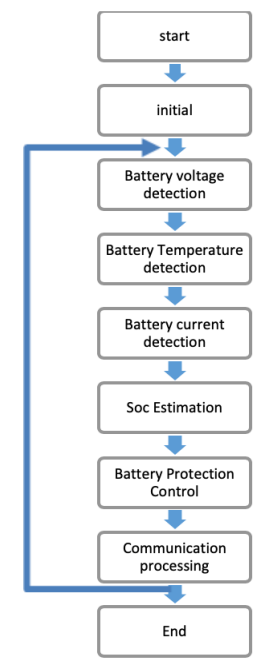

Fig. 6. The main program flowchart of the centralized control unit
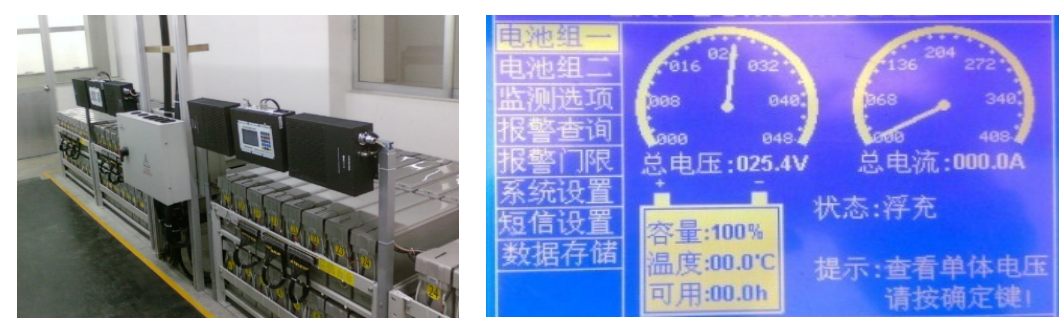

Fig. 7. The photos of the centralized control unit(Left picture) and the real-time display interface diagram of centralized control unit(Right picture)

\section{The remote monitoring center}

The battery online monitoring system collects various parameters of the battery pack, and uses the internet network to transmit and transmit data in real time, and centrally store it in the data receiving server of the remote monitoring center, and perform data analysis and statistics through the application program. Providing a friendly human-computer interaction management interface, the operation and maintenance personnel can view the real-time operation information and historical operation data of each substation battery pack through the IE browser, and realize online monitoring and management of energy strorage battery.

People can conveniently check the total voltage, total current, cell voltage, cell temperature of each battery cell in real time through the remote monitoring center. The total voltage, total current, cell voltage, temperature and other graphs, discharging characteristic curve, voltage bar graph, capacity analysis table, etc. can also be displayed on monitering interface, as shown in Figure 8. The battery-related data of the remote monitoring center is convenient for people to view, manage and save. 


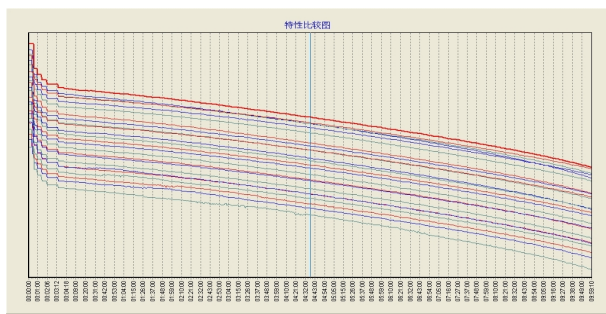

A. Discharge characteristic curve of energy storage battery

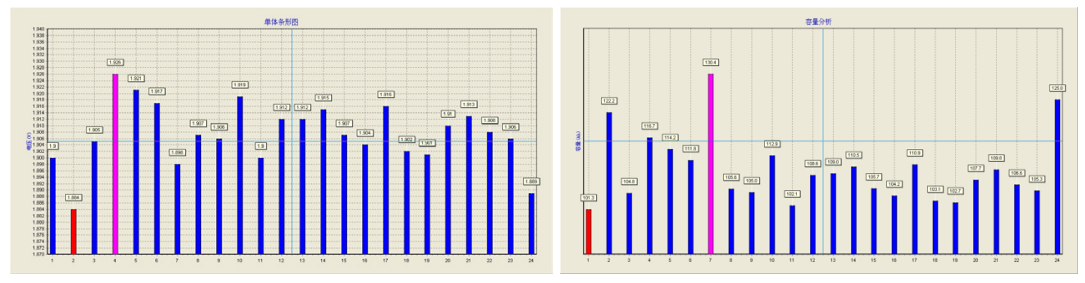

B. Voltage and capacity histogram of energy storage battery

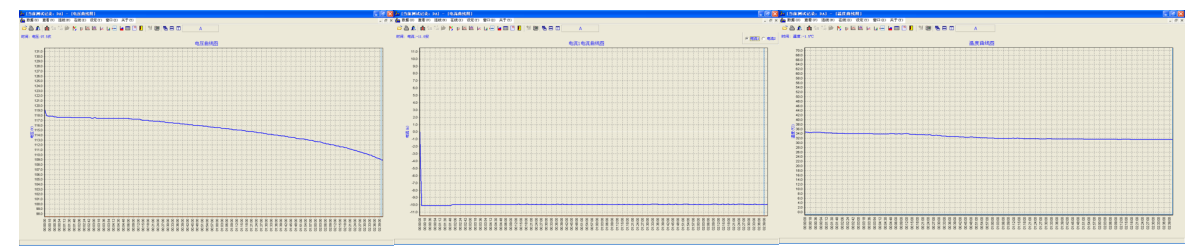

C. Voltage, current and temperature graph of energy storage battery cells

Fig. 8. Various data graphs for online monitoring on battery status (A. Discharge characteristic curve of energy storage battery, B. Voltage and capacity histogram of energy storage battery, C. Voltage, current and temperature graph of energy storage battery cells)

\section{Application of energy storage battery online monitoring system}

In order to verify the measurement accuracy of the online monitoring system under normal working temperature, a set of lithium manganate batteries and high-precision adjustable analog batteries are used as test objects, and the actual voltage of the battery is measured by a high precision multimeter, and the online monitoring is performed. The voltage difference measured by the system results in a voltage error, as shown in Table 1. It can be seen from Table 1 that the measurement error of the measurement module is between $\pm 1.9 \mathrm{mV}$ at normal temperature, the error is very small, and the measurement accuracy of the online monitoring data is high. 
Table 1. Voltage detection error of energy storage battery online monitoring system

\begin{tabular}{|c|c|c|c|c|}
\hline Battery No. & Actual voltage/V & Detection voltage/V & Voltage error/mV & $\begin{array}{c}\text { Percentage of voltage er- } \\
\text { ror/\% }\end{array}$ \\
\hline 1 & 3.6585 & 3.657 & -1.5 & -0.041 \\
\hline 2 & 3.6666 & 3.668 & 1.4 & 0.038183 \\
\hline 3 & 3.6599 & 3.661 & 1.1 & 0.030055 \\
\hline 4 & 3.6709 & 3.669 & -1.9 & -0.05176 \\
\hline 5 & 3.6816 & 3.682 & 0.4 & 0.010865 \\
\hline 6 & 3.6316 & 3.632 & 0.4 & 0.011014 \\
\hline
\end{tabular}

After the actual application test, the online monitoring system has good battery state detection accuracy, which can meet the requirements of online battery monitoring. The specific technical parameters are shown in Table 2.

Table 2. Measuring range and accuracy of energy storage battery online monitoring system

\begin{tabular}{|l|c|c|}
\hline Measuring object & Measuring range & Measuring accuracy \\
\hline \multirow{3}{*}{ Voltage of battery pack } & $0 \sim 150 \mathrm{~V}$ & $0.2 \% \pm 0.1 \mathrm{~V}$ \\
\cline { 2 - 3 } & $0 \sim 300 \mathrm{~V}$ & $0.3 \% \pm 0.1 \mathrm{~V}$ \\
\cline { 2 - 3 } & $0 \sim 600 \mathrm{~V}$ & $0.3 \% \pm 0.2 \mathrm{~V}$ \\
\hline \multirow{3}{*}{ Voltage of battery cell } & $0 \sim 5 \mathrm{~V}$ & $0.1 \% \pm 0.5 \mathrm{mV}$ \\
\cline { 2 - 3 } & $0 \sim 15 \mathrm{~V}$ & $0.1 \% \pm 1 \mathrm{mV}$ \\
\hline \multirow{3}{*}{$\begin{array}{l}\text { Current of charging and dis- } \\
\text { charging }\end{array}$} & $0 \sim 45 \mathrm{~V}$ & $0.1 \% \pm 10 \mathrm{mV}$ \\
\cline { 2 - 3 } & $0 \sim 10 \mathrm{~A}$ & $0.5 \% \pm 0.1 \mathrm{~A}$ \\
\cline { 2 - 3 } & $0 \sim 50 \mathrm{~A}$ & $1 \% \pm 0.2 \mathrm{~A}$ \\
\cline { 2 - 3 } & $0 \sim 100 \mathrm{~A}$ & $1 \% \pm 0.5 \mathrm{~A}$ \\
\hline
\end{tabular}

\section{Conclusion}

The online monitoring system for energy storage lithium-ion batteries is designed in this paper. Based on the $\mathrm{B} / \mathrm{S}$ network structure, it consists of a battery state detection unit, a centralized control unit and a remote monitoring center. The online monitoring system monitors and analyzes the battery real-time status data, accurately calculates the battery SOC, realizes the online real-time monitoring of the lithium ion battery, prevents the lithium ion battery from overcharging, over-discharging, overheating, and promotes the safe and stable operation of the lithium-ion energy storage battery. The lithium ion battery online monitoring system has the advantages of safety, reliability, and high precision, and has been successfully applied to the energy storage battery management task of distributed generation. 


\section{Acknowledgment}

Author's research project has accepted funding from the Nature Science Fund of Shandong Province (the program NO. is ZR2015EL041) and Heze University Nature Science Fund (the program NO. is XY14KJ06).

\section{References}

[1] Zhai Haizhou. Modeling of Lithium-ion Battery for Charging/Discharging Characteristics Based on Circuit Model. International Journal of Online Engineering, 2017(13)6, 86-95.

[2] Zhang Wei,Yang Jingfa and Yan Qigeng,"Experiment research on the property of silicon solar cell", Experimental Technology and Management.vol.26, no.9, pp. 42-46, 2009.

[3] Shariff, F., Rahim, N. A., \& Hew, W. P. (2015). Zigbee-based data acquisition system for online monitoring of grid-connected photovoltaic system. Expert Systems with Applications, 42(3), 1730-1742. https://doi.org/10.1016/j.eswa.2014.10.007

[4] Lars Johannesson, Stefan Pettersson, Bo Egardt. Predictive energy management of a 4QT series-parallel hybrid electric bus. Control Engineering Practice. 2009 (12). 886 896. https://doi.org/10.1016/j.conengprac.2009.07.004

[5] M. W. Verbrugge, R. S. Conell. Electrochemical and thermal characterization of battery modules commensurate with electric vehicle integration. Journal of the Electrochemical Society, 2002, 149(1). A45 A53. https://doi.org/10.1149/1.1426395

[6] B. S. Bhangu, P. Bentley, D. A. Stone, C. M. Bingham. Observer techniques for estimating the State-of-Charge and State-of-Health of VRLABs for Hybrid Electric Vehicles. IEEE Conf. Vehicle Power and Propulsion. 2005. 780 789. https://doi.org/10.1109/vppc.2005. 1554646

[7] Gui, L., Ruan, T. Y., Wang, Z. Z., Sun, A. C., \& Xu, M. (2015). Cnc online monitoring system based on internet of things. Advanced Materials Research, 1079-1080(6), 672-678.

[8] Zhang, F., Liu, M., Zhou, Z., \& Shen, W. (2016). An iot-based online monitoring system for continuous steel casting. IEEE Internet of Things Journal, 3(6), 1355-1363. https://doi.org/10.1109/JIOT.2016.2600630

[9] Yang, X., Luo, H., Krueger, M., Ding, S. X., \& Peng, K. (2016). Online monitoring system design for roll eccentricity in rolling mills. IEEE Transactions on Industrial Electronics, 63(4), 2559-2568. https://doi.org/10.1109/TIE.2015.2442223

\section{Author}

Zhai Haizhou is associated professor of Heze University, Heze City, Shandong Province, PR China. His works focus on electrode materials and control system of liion batteries.

Article submitted 17 August 2018. Final acceptance 15 November 2018. Final version published as submitted by the author. 